\title{
First-principles calculations of a high-pressure synthesized compound PtC
}

\author{
Linyan $\mathbf{L i}^{1}$, Wen $\mathbf{Y u}^{1,2}$ and Changqing $\mathbf{J i n}^{1,3}$ \\ ${ }^{1}$ Institute of Physics, Chinese Academy of Sciences, PO Box 603, Beijing 100080, \\ People's Republic of China \\ 2 Physics Department, University of Science and Technology Beijing, Beijing 100080, \\ People's Republic of China \\ E-mail: cqjin@aphy.iphy.ac.cn
}

Received 21 July 2005, in final form 19 August 2005

Published 9 September 2005

Online at stacks.iop.org/JPhysCM/17/5965

\begin{abstract}
The first-principles density-functional method is used to study the recently high-pressure synthesized compound PtC. It is confirmed by our calculations that platinum carbide has a zinc-blende ground-state phase at zero pressure and that the rock-salt structure is a high-pressure phase. The theoretical transition pressure from zinc-blende to rock-salt structure is determined to be $52 \mathrm{GPa}$. Furthermore, our calculation shows the possibility that the PtC experimentally synthesized under high pressure conditions might undergo a transition from rock-salt to zinc-blende structure after a pressure quench to ambient conditions.
\end{abstract}

\section{Introduction}

Some carbides, especially transition-metal carbides, are widely used for industrial application because of their remarkable physical properties. In a recent paper, Ono et al [1] synthesized a new platinum carbide. The compound is formed at high temperature and high pressure and was found to be stable after quenching to room temperature and ambient pressure. They thought that the new PtC has a cubic symmetry and a rock-salt type structure with space group $F m 3 m$ by analysing the synchrotron x-ray diffraction pattern. But it is impossible to distinguish between the zinc-blende (space-group number 216) and rock-salt (space-group number 225) structures solely from the X-ray diffraction because of the large mass difference between $\mathrm{Pt}$ and C. In this case, the Raman spectrum is an important criterion. For example, Gregoryanz et al [2] synthesized PtN at high temperature and high pressure, and the crystal structure of $\mathrm{PtN}$ has a cubic symmetry from the X-ray diffraction. They concluded that $\mathrm{PtN}$ has a zincblende structure because the rock-salt structure does not have a first-order Raman spectrum. In this study, we investigated the relative stability of the two different structures for PtC and we discuss the possible phase transitions under pressure from a theoretical point of view.

3 Author to whom any correspondence should be addressed. 


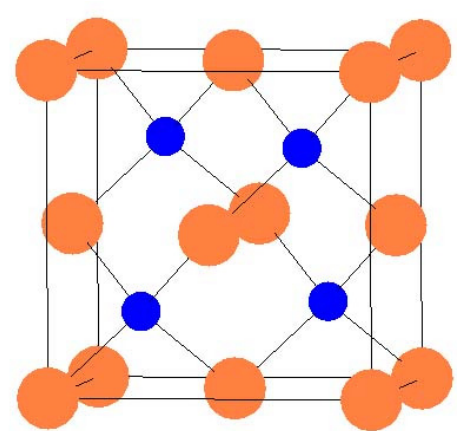

(a)

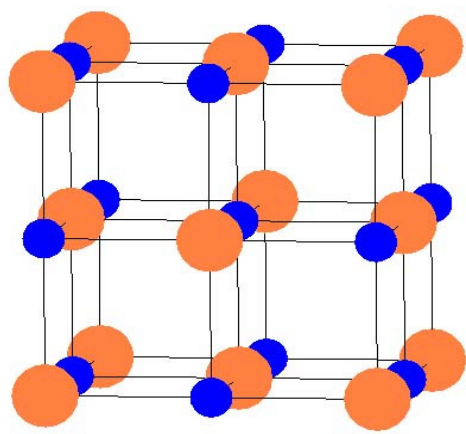

(b)

Figure 1. Unit-cell schematics of (a) the zinc-blende structure and (b) the rock-salt structure. The large and small spheres denote $\mathrm{Pt}$ and $\mathrm{C}$ atoms, respectively.

(This figure is in colour only in the electronic version)

\section{Method of calculation}

We used first-principles method to calculate the zinc-blende and rock-salt structures stability of $\mathrm{PtC}$ as a function of pressure. All calculations were performed using the Vienna package WIEN2k [3]. This is a full-potential linearized augmented plane waves (LAPW) method within the density-functional theory (DFT) [4]. This method is one of the most accurate schemes for solving the Kohn-Sham equations. The generalized gradient approximation (GGA) [5] was used for the exchange and correlation potential function. In the zinc-blende structure, muffintin (MT) sphere radii of 2.2 and 1.5 bohr were used for $\mathrm{Pt}$ and $\mathrm{C}$ atoms respectively, while in the rock-salt structure 2.3 and 1.7 bohr were used because the lattice constants of the two structures are different. The valence wavefunctions inside the MT spheres were expanded into spherical harmonics up to $l=10$ and the $R_{\mathrm{mt}} K_{\max }$ were taken to be 8.0. We used $3000 k$-points in the Brillouin zone for the zinc-blende structure and $4000 k$-points for the rock-salt structure. The self-consistent calculations were considered to be converged only when the integrated magnitude of the charge density difference between input and output (i.e., $\left.\int\left|\rho_{n+1}(\vec{r})-\rho_{n}(\vec{r})\right| \mathrm{d} \vec{r}\right)$ was less than 0.00001 .

\section{Results and discussion}

\subsection{Structural properties}

The zinc-blende and rock-salt structures are shown in figure 1. In the zinc-blende structure, the Pt atoms form a face-centred cubic lattice and the $\mathrm{C}$ atoms occupy half of the tetrahedral interstitial sites of the Pt lattice. In the rock-salt structure, the two face-centred cubic lattices formed respectively by $\mathrm{Pt}$ and $\mathrm{C}$ atoms are interpenetrated. Figure 2 is a plot of total energy as a function of unit-cell volume for the two structures. It shows that the zinc-blende structure for $\mathrm{PtC}$ has the lower energy minimum and that the minimum occurs at a larger lattice constant than for the rock-salt structure. This means that the zinc-blende structure is the ground-state phase at zero pressure and at a sufficiently high pressure the rock-salt structure would be favoured. The structural phase transition would occur at a pressure where the Gibbs free energies of the two structures are the same. Since all our calculations are performed at $T=0 \mathrm{~K}$, then the Gibbs free energy, $G=E_{0}+P V-T S$, becomes equal to the enthalpy, $H=E_{0}+P V$. 


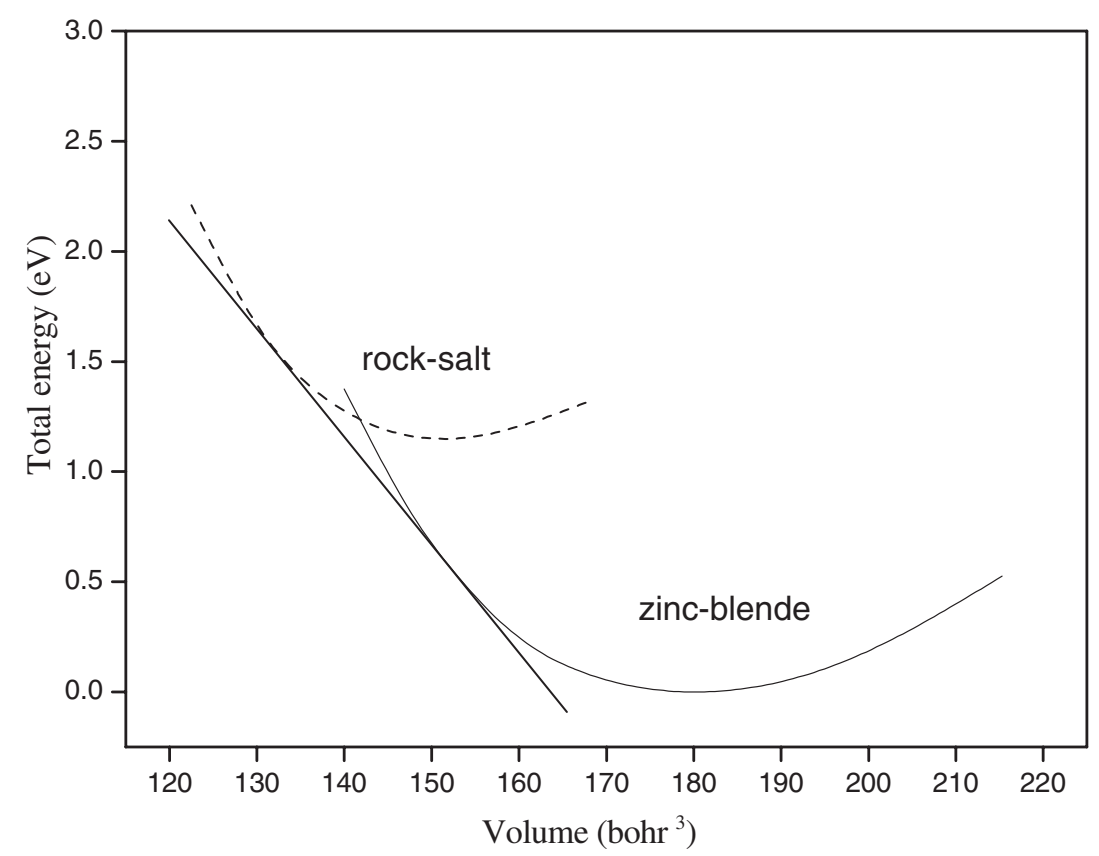

Figure 2. Total energy as a function of the volume per formula unit for $\mathrm{PtC}$ in zinc-blende (solid line) and the rock-salt (dashed line) structures.

Table 1. Lattice constants, bulk modulus, and total energy with respect to the $\mathrm{PtC}$ in the zinc-blende and rock-salt structures.

\begin{tabular}{llll}
\hline & Exp. & Zinc-blende (cal.) & Rock-salt (cal.) \\
\hline$a(\AA)$ & 4.814 & 4.737 & 4.494 \\
$B(\mathrm{GPa})$ & 301 & 230 & 257 \\
$E(\mathrm{eV})$ & & -1.17 & 0.0 \\
\hline
\end{tabular}

Therefore, the phase transition pressure is determined with the tangent to the curves in figure 2 . The slope of the tangent line reveals that, at a pressure of about $52 \mathrm{GPa}$, a phase transition from zinc-blende to rock-salt structure would occur if the temperature were high enough to break the zinc-blende covalent bonds.

The lattice constants, bulk modulus, and total energies of the two structures for PtC are listed in table 1 . The bulk modulus $(B)$ was evaluated from the Murnaghan equation fitting of the total energies as a function of the unit-cell volume. The theoretical zinc-blende lattice constant is in agreement with experiment while the rock-salt lattice constant is $7 \%$ smaller than the experimental value of the quenched $\mathrm{PtC}$ sample. The total energy per formula unit of the zinc-blende structure is about $1.17 \mathrm{eV}$ smaller than that of the rock-salt structure.

\subsection{Elastic properties}

In order to further confirm the structure stability under strain, we calculated the elastic constants of the two structures, which are shown in table 2 . The mechanical stability of a crystal means that the strain energy must be positive. There are three independent elastic constants, $c_{11}, c_{12}$ and $c_{44}$ for a cubic crystal. A stable cubic crystal should match with the conditions [6]: 


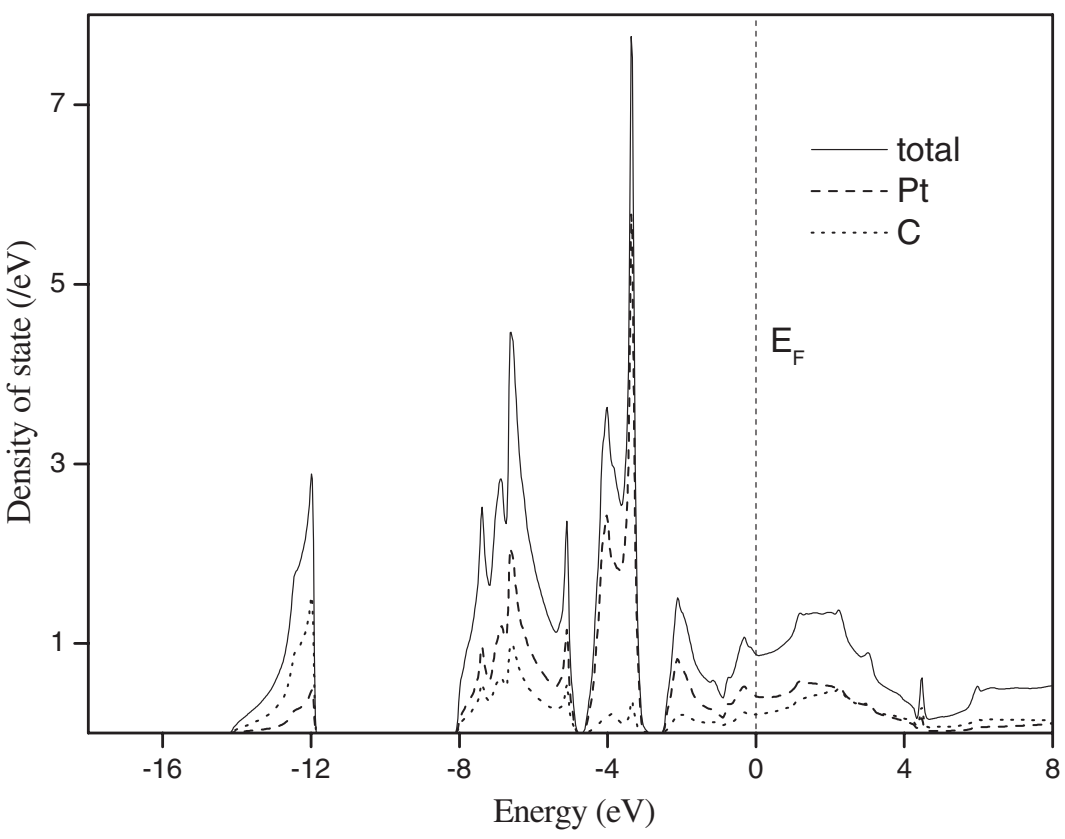

Figure 3. Total and partial densities of state of PtC in the zinc-blende structure.

Table 2. Elastic constants of $\mathrm{PtC}$ in the zinc-blende and rock-salt structures.

\begin{tabular}{llllll}
\hline & $\begin{array}{l}c_{11} \\
(\mathrm{GPa})\end{array}$ & $\begin{array}{l}c_{12} \\
(\mathrm{GPa})\end{array}$ & $\begin{array}{l}c_{44} \\
(\mathrm{GPa})\end{array}$ & $\begin{array}{l}B=\left(c_{11}+2 c_{12}\right) / 3 \\
(\mathrm{GPa})\end{array}$ & $c_{11}-\left|c_{12}\right|$ \\
\hline Zinc-blende & 284 & 199 & 70 & 227 & $>0$ \\
Rock-salt & 252 & 271 & 55 & 265 & $<0$ \\
\hline
\end{tabular}

$c_{44}>0, c_{11}>\left|c_{12}\right|$ and $c_{11}+2 c_{12}>0$. The three elastic constants can be obtained from the second-order derivative of the total energy of the crystal under three types of shear strains: the volume change, the volume conserved tetragonal and rhombohedral strains. The bulk modulus evaluated from the elastic constants (table 2) were consistent with those obtained from the Murnaghan equation (table 1) for the two structures of PtC. The rock-salt bulk modulus we calculated from the Murnaghan equation is $265 \mathrm{GPa}$, which is about $12 \%$ smaller than the experimental value of $301 \mathrm{GPa}$. In the rock-salt structure, $c_{11}<\left|c_{12}\right|$, and in the zinc-blende structure, the restrictions are all fulfilled. With these results, we conclude that the zinc-blende structure is the stable ground state and the rock-salt structure is not stable at zero pressure, and there is a possibility that a phase transition from rock-salt (high-pressure phase) to zinc-blende (ground-state phase) would occur after a pressure quench to ambient conditions.

\subsection{Electronic properties}

Figure 3 displays the total and partial densities of states (DOS) for the zinc-blende PtC. The rock-salt DOS is similar to that of zinc-blende. No energy gap is seen, indicating a metallic nature of platinum carbide. At the Fermi level the total DOS is 0.88 states/eV unit cell. The DOS of $\mathrm{Pt}$ is 0.41 states $/ \mathrm{eV}$ unit cell and that of $\mathrm{C}$ is 0.21 . It is evident that the main DOS is from the $\mathrm{Pt}(\mathrm{Pt} 5 \mathrm{~d})$ contribution. The states between -14 and $-11 \mathrm{eV}$ are mainly composed of $\mathrm{C}(2 \mathrm{~s})$ states. The states above $-8 \mathrm{eV}$ are mainly composed of $\mathrm{Pt}(5 \mathrm{~d})$ states and C (2p) states. 

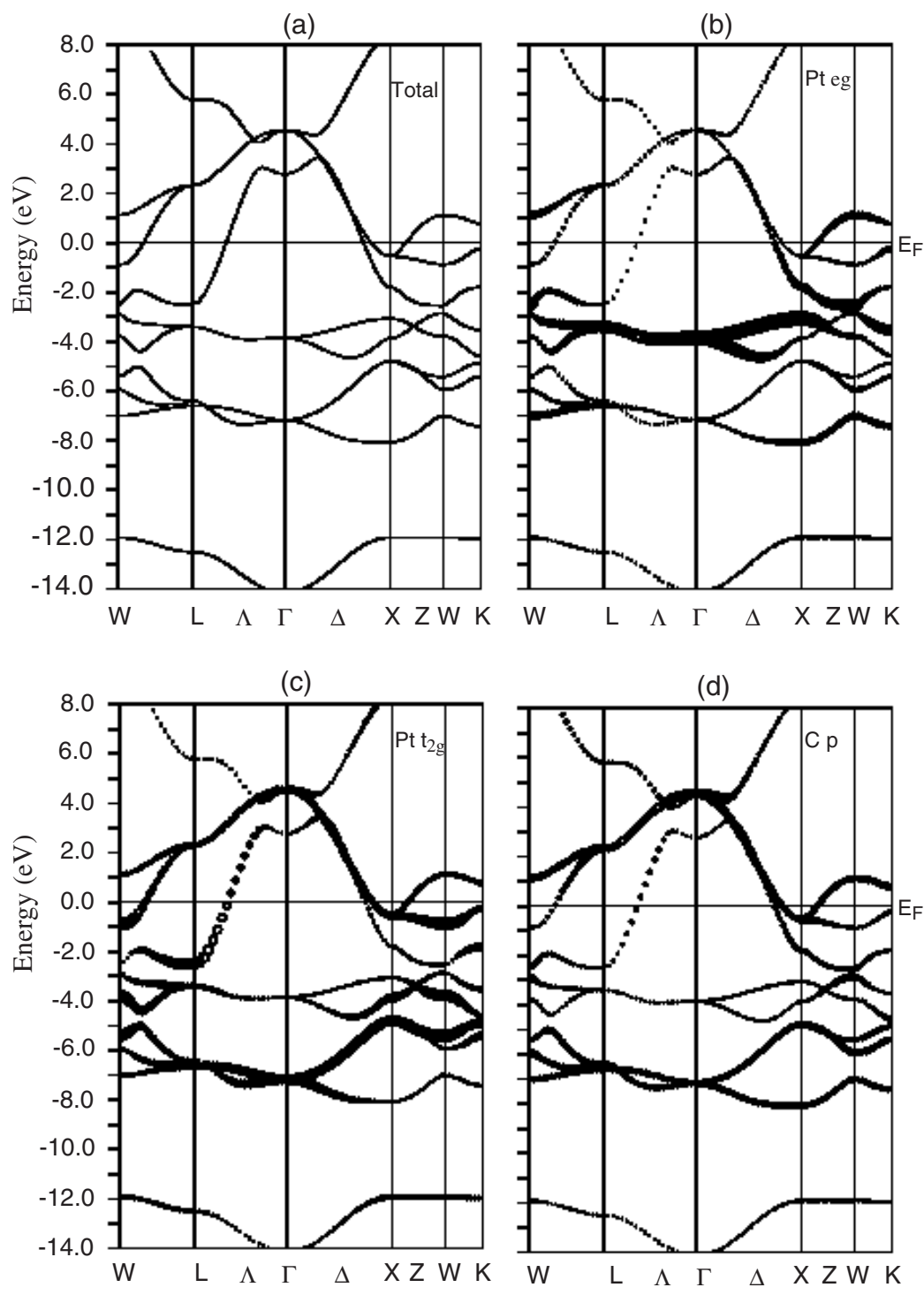

Figure 4. Energy bands and characteristic bands of zinc-blende PtC along high-symmetry directions near the Fermi energy (located at $E=0$ ).

Due to the stronger $\mathrm{Pt}\left(\mathrm{t}_{2 \mathrm{~g}}\right)-\mathrm{C}(2 \mathrm{p})$ interactions, the $\mathrm{t}_{2 \mathrm{~g}}$ band has a larger dispersion than that of the $e_{\mathrm{g}}$ band.

Figure 4 displays the energy bands and the characteristic bands along high-symmetry directions in the Brillouin zone. The band around $-12 \mathrm{eV}$ originates almost completely from $\mathrm{C} 2 \mathrm{~s}$ states. The bands around -3 and $-4 \mathrm{eV}$ originate mainly from $\mathrm{Pt} \mathrm{e}_{\mathrm{g}}$ states. From -7 to $1 \mathrm{eV}$, the bands are a hybridization between $\mathrm{Pt} \mathrm{t}_{2 \mathrm{~g}}$ and $\mathrm{C} 2 \mathrm{p}$. From the characteristic bands of $\mathrm{Pt}$ $\mathrm{e}_{\mathrm{g}}, \mathrm{Pt}_{2 \mathrm{~g}}$ and $\mathrm{C} 2 \mathrm{p}$, the major contribution to the covalent bonds comes from the hybridization between Pt $5 \mathrm{~d}$ and $\mathrm{C} 2 \mathrm{p}$. 


\section{Conclusions}

In summary, we have studied PtC in the zinc-blende and rock-salt structures using firstprinciples calculations. With all the calculations we conclude that the zinc-blende structure of $\mathrm{PtC}$ is the ground-state phase at zero pressure while the rock-salt structure is a high-pressure phase. At a pressure of about $52 \mathrm{GPa}$, a phase transition from zinc-blende to rock-salt structure may occur. Our calculation also shows the possibility that the PtC experimentally synthesized by Ono et al under high-pressure conditions might undergo a transition from rock-salt to zinc-blende structure after a pressure quench to ambient conditions.

\section{Acknowledgment}

This work was partially supported by the National Nature Science Foundation of China, the State Key Fundamental Research Project (2002CB613301).

\section{References}

[1] Ono S, Kikegawa T and Ohishi Y 2005 Solid State Commun. 133 55-9

[2] Gregoryanz B, Sanloup C, Somayazulu M, Badro J, Fiquet G, Mao H-K and Hemley R J 2004 Nat. Mater. 3294

[3] Blaha P, Schwarz K, Sorantin P and Trickey S B 1990 Comput. Phys. Commun. 59399

[4] Hohenberg P and Kohn W 1964 Phys. Rev. 136 B864 Kohn W and Sham L J 1965 Phys. Rev. 140 A1133

[5] Perdew J P, Burke K and Ernzerhof M 1996 Phys. Rev. Lett. 773865

[6] Nye J F 1985 Physical Properties of Crystals (Oxford: Oxford University Press) 